\title{
Precipitation in Gallipoli: Sugammadex / Amiodarone \& Sugammadex / Dobutamine \& Sugammadex / Protamine
}

Dear Editor,

Sugammadex is a modified gamma cyclodextrin ${ }^{1-3}$. Cyclodextrins are water soluble cyclic oligosaccharides with a lipophilic core. Sugammadex has quickly found a place in clinical use for selective antagonism of neuromuscular blockade with rocuronium $^{1-3}$. Sugammadex quickly encapsulates steroidal neuromuscular blockers, increasing the amount of encapsulated steroidal neuromuscular blockers in plasma and separating the blockers from the nicotinic acetylcholine receptors ${ }^{1-3}$.

Apart from its use with steroidal neuromuscular blockers, it is known that sugammadex interacts with over 40 lipophilic, steroidal and non-steroidal drugs. These drugs include: propofol, thiopental, fentanyl, remifentanil, vancomycin, gentamicin, salbutamol, aminophylline, atropine, digoxin, ephedrine, phentolamine, verapamil, cortisone and hydrocortisone ${ }^{4}$.

Previous research shows that, apart from steroidal muscle relaxants, sugammadex forms precipitates with protamine ${ }^{5}$.

There is no clinical data on whether sugammadex forms precipitates with other drugs, commonly used in clinical practice. The hypothesis of this study is to investigate whether the chemical and physical properties of sugammadex would contribute to a precipitation reaction with drugs commonly used in clinical practice.

With this aim, simply $0.1 \mathrm{~mL}$ containing $100 \mathrm{mg} \cdot \mathrm{mL}^{-1}$ sugammadex was mixed on a glass slide with the same volume of a drug and the presence or absence of precipitation was determined under the microscope. The drugs investigated are all commonly used in clinical anesthesia practice. They include: adrenalin $\left(1 \mathrm{mg} \cdot \mathrm{mL}^{-1}\right)$, atropine $\left(1 \mathrm{mg} \cdot \mathrm{mL}^{-1}\right)$, amiodarone $\left(50 \mathrm{mg} \cdot \mathrm{mL}^{-1}\right)$, aminophylline $\left(24 \mathrm{mg} \cdot \mathrm{mL}^{-1}\right)$, ornidazole (500 mg. $\left.3 \mathrm{~mL}^{-1}\right)$, bupivacaine $\left(5 \mathrm{mg} \cdot \mathrm{mL}^{-1}\right)$, cefazolin sodium (250 mg. mL $\left.{ }^{-1}\right)$, dexmedetomine $\left(100 \mu \mathrm{g} \cdot \mathrm{mL}^{-1}\right)$, dobutamine (12.5 mg. $\left.\mathrm{mL}^{-1}\right)$, dopamine $\left(8 \mathrm{mg} \cdot \mathrm{mL}^{-1}\right)$, ephedrine $\left(0.05 \mathrm{~g} \cdot \mathrm{mL}^{-1}\right)$, esmolol (10 mg. $\left.\mathrm{mL}^{-1}\right)$, esomeprazole $\left(40 \mathrm{mg} \cdot \mathrm{mL}^{-1}\right)$, etomidate (2 mg. $\left.\mathrm{mL}^{-1}\right)$, fentanyl $\left(50 \mu \mathrm{g} \cdot \mathrm{mL}^{-1}\right)$, flumazenil $\left(0.1 \mathrm{mg} \cdot \mathrm{mL}^{-1}\right)$, furosemide $\left(10 \mathrm{mg} \cdot \mathrm{mL}^{-1}\right)$, gentamicin $\left(40 \mathrm{mg} \cdot \mathrm{mL}^{-1}\right)$, glyceryl trinitrate $\left(5 \mathrm{mg} \cdot \mathrm{mL}^{-1}\right)$, heparin $\left(1,000 \mathrm{IU} \cdot \mathrm{mL}^{-1}\right)$, hydrocortisone $\left(250 \mathrm{mg} \cdot \mathrm{mL}^{-1}\right)$, crystallized insulin $\left(100 \mathrm{IU} \cdot \mathrm{mL}^{-1}\right)$, Calcium (Calcium Gluconate Monohydrate $225 \mathrm{mg} .10 \mathrm{~mL}^{-1}$ + Calcium levulinate dihitrate $572 \mathrm{mg} .10 \mathrm{~mL}^{-1}$ ), ketamine (50 mg. $\left.\mathrm{mL}^{-1}\right)$, levobupivacaine $\left(7.5 \mathrm{mg} \cdot \mathrm{mL}^{-1}\right)$, magnesium sulphate (1.2 mEq. $\left.\mathrm{mL}^{-1}\right)$, metamizol sodium $\left(0.5 \mathrm{~g} \cdot \mathrm{mL}^{-1}\right)$, methylergobasin maleate $\left(0.2 \mathrm{mg} \cdot \mathrm{mL}^{-1}\right)$, metoclopramide (5 mg. $\left.\mathrm{mL}^{-1}\right)$, metoprolol (1 mg. $\left.\mathrm{mL}^{-1}\right)$, morphine $\left(0.01 \mathrm{~g} \cdot \mathrm{mL}^{-1}\right)$, midazolam (5 mg. $\left.\mathrm{mL}^{-1}\right)$, n-acetylcysteine $\left(100 \mathrm{mg} \cdot \mathrm{mL}^{-1}\right)$, naloxone $\left(0.4 \mathrm{mg} \cdot \mathrm{mL}^{-1}\right)$, neostigmine $\left(0.5 \mathrm{mg} \cdot \mathrm{mL}^{-1}\right)$, nitroprusside (12 mg. $\left.\mathrm{mL}^{-1}\right)$, noradrenaline (1 mg. $\left.\mathrm{mL}^{-1}\right)$, oxytocin $\left(5 \mathrm{lU} \cdot \mathrm{mL}^{-1}\right)$, paracetamol $\left(10 \mathrm{mg} \cdot \mathrm{mL}^{-1}\right)$, thiopental sodium $\left(25 \mathrm{mg} \cdot \mathrm{mL}^{-1}\right)$, pethidine $\left(50 \mathrm{mg} \cdot \mathrm{mL}^{-1}\right)$, pheniramine $\left(22.75 \mathrm{mg} \cdot \mathrm{mL}^{-1}\right)$, phenytoin ( $\left.50 \mathrm{mg} \cdot \mathrm{mL}^{-1}\right)$, piracetam $\left(1 \mathrm{~g} .5 \mathrm{~mL}^{-1}\right)$, prednisolone (25 mg), prilocaine $\left(20 \mathrm{mg} \cdot \mathrm{mL}^{-1}\right)$, propafenone $\left(3.5 \mathrm{mg} \cdot \mathrm{mL}^{-1}\right)$, protamine hydrochloride $\left(1,000 \mathrm{IU} \cdot \mathrm{mL}^{-1}\right)$, potassium $\left(1 \mathrm{mEq} \cdot \mathrm{mL}^{-1}\right)$, remifentanil (5 mg. $\left.\mathrm{mL}^{-1}\right)$, cefuroxime axetil $\left(125 \mathrm{mg} \cdot \mathrm{mL}^{-1}\right)$, sulbactam-ampicillin (200 mg. $\left.\mathrm{mL}^{-1}\right)$, succinylcholine (20 mg. $\left.\mathrm{mL}^{-1}\right)$, tenoxicam $\left(10 \mathrm{mg} \cdot \mathrm{mL}^{-1}\right)$, theophylline $(24 \mathrm{mg}$. $\left.\mathrm{mL}^{-1}\right)$, tramadol (50 mg. $\left.\mathrm{mL}^{-1}\right)$, and vitamin $\mathrm{K}\left(10 \mathrm{mg} \cdot \mathrm{mL}^{-1}\right)$. A scale of $0-4$ was used to evaluate the test, with 0 being no precipitation and 4+ being strong precipitation.

Within seconds, sugammadex $\left(100 \mathrm{mg} \cdot \mathrm{mL}^{-1}\right)$ scored 4+ precipitation with amidarone (50 mg. $\left.\mathrm{mL}^{-1}\right)$, dobutamine $(12.5 \mathrm{mg}$. $\left.\mathrm{mL}^{-1}\right)$ and protamine hydrochloride $\left(1,000 \mathrm{IU} \cdot \mathrm{mL}^{-1}\right)$.

There are reports on the incompatibility and precipitation of anesthetic drugs ${ }^{5-7}$. Thiopental, with basic $\mathrm{pH}$, reacts with acidic muscle relaxants such as suxamethonium, mivacurium, vecuronium and rocuronium, causing precipitation ${ }^{8}$. Similarly, thiopental causes precipitation with local anesthetics due to $\mathrm{pH}$ differences ${ }^{9}$. Previous experimental studies have shown that thiopental reacting with various drugs may form thiopental acid crystals due to precipitation, which may cause pulmonary embolism ${ }^{10}$. Sugammadex has a pH of 7.5 and the precipitation reaction with protamine may be related to the molecules' ionic weight ${ }^{1-5}$. 
Our study shows that sugammadex reacts with amiodarone, dobutamine and protamine under in-vitro conditions, causing precipitation. We emphasize that sugammadex should not be given intravenously simultaneously with these drugs. Future studies will characterize their precipitation reaction, which seems to be only the tip of the iceberg. In addition, future studies should be focused on investigating sugammadex compatibility with other drugs by using Gas Chromatography/Mass Spectrometry device. We believe the effects of this precipitation on the drugs' efficacy and circulation warrants further research.

*Volkan Hanci, MD, Assoc. Prof. **Hasan Ali Kiraz, MD, Assist. Prof. **Dilek Ömür, MD, Assist. Prof. **Serpil Ekin, MD, Assistant Doctor **Berna Uyan, MD, Assistant Doctor ***Bulent Serhan Yurtlu, MD, Assist. Prof.

Departments of Anaesthesiology and Reanimation, * Dokuz Eylul University (formerly Canakkale Onsekiz Mart University)

** Canakkale Onsekiz Mart University

*** Dokuz Eylul University (formerly Bulent Ecevit University), Turkey

\section{References}

1. Naguib $M$ - Sugammadex: another milestone in clinical neuromuscular pharmacology. Anesth Analg, 2007;104:575-581.

2. Brull SJ, Naguib M - Selective reversal of muscle relaxation in general anesthesia: focus on sugammadex. Drug Des Devel Ther, 2009;3:119-129.

3. Rex C, Bergner UA, Pühringer FK - Sugammadex: a selective relaxant-binding agent providing rapid reversal. Curr Opin Anaesthesiol, 2010;23:461-465.

4. Zhang $M-Q$ - Drug-specific cyclodextrins: the future of rapid neuromuscular block reversal? Drugs Future. 2003;28:347-354.

5. Alston TA - Precipitation of sugammadex by protamine. J Clin Anesth, 2011;23:593.

6. Smith RP, Jones M - Precipitation in Manchester: ketorolac/ cyclizine. Anaesthesia, 2001;56:494-495.

7. Ackland G - Physical incompatibility between atracurium and intravenous diclofenac. Anaesthesia, 2001;56:294.

8. Khan S, Stannard N, Greijn J - Precipitation of thiopental with muscle relaxants: a potential hazard. JRSM Short Rep, 2011;2:58.

9. Waters $\mathrm{JH}$, Rizzo VL, Ramanathan $\mathrm{S}$ - A re-evaluation of the ability of thiopental to identify cerebrospinal fluid in epidural catheter aspirate. J Clin Anesth, 1995;7:224-227.

10. Taniguchi T, Yamamoto K, Kobayashi T - Precipitate formed by thiopentone and vecuronium causes pulmonary embolism. Can J Anaesth, 1998;45:347-351. 